Article

\title{
Current Food Consumption amongst the Spanish ANIBES Study Population
}

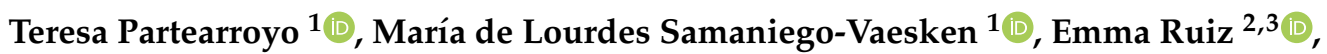

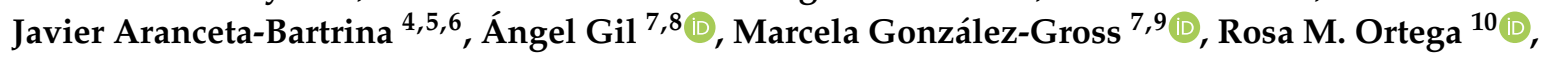 \\ Lluis Serra-Majem ${ }^{5,7,11,12}$ (D) and Gregorio Varela-Moreiras 1,13,*
}

1 Departamento de Ciencias Farmacéuticas y de la Salud, Facultad de Farmacia, Universidad San Pablo-CEU, CEU Universities, Urbanización Montepríncipe, 28925 Alcorcón, Madrid, Spain; t.partearroyo@ceu.es (T.P.); 1.samaniego@ceu.es (M.d.L.S.-V.)

2 CIBERESP, Consortium for Biomedical Research in Epidemiology and Public Health, Carlos III Health Institute, 28029 Madrid, Spain; e.ruiz@externos.isciii.es

3 National Center for Epidemiology, Carlos III Health Institute, 28029 Madrid, Spain

4 Department of Food Sciences and Physiology, University of Navarra, Pamplona, 31009 Navarra, Spain; javieraranceta@gmail.com

5 Research Institute of Biomedical and Health Sciences, University of Las Palmas de Gran Canaria, 35016 Las Palmas, Spain; 1luis.serra@ulpgc.es

6 Department of Physiology, Faculty of Medicine, University of the Basque Country (UPV/EHU), 48940 Leioa, Vizcaya, Spain

7 CIBEROBN, Biomedical Research Networking Center for Physiopathology of Obesity and Nutrition, Carlos III Health Institute, 28029 Madrid, Spain; agil@ugr.es (Á.G.); marcela.gonzalez.gross@upm.es (M.G.-G.)

8 Department of Biochemistry and Molecular Biology II and Institute of Nutrition and Food Sciences, University of Granada, 18010 Granada, Spain

9 ImFINE Research Group, Department of Health and Human Performance, Universidad Politécnica de Madrid, 28040 Madrid, Spain

10 Department of Nutrition and Food Science, Faculty of Pharmacy, Madrid Complutense University, 28040 Madrid, Spain; rortega@ucm.es

11 Research Institute of Biomedical and Health Sciences, University of Las Palmas de Gran Canaria, 35016 Las Palmas, Spain

12 Service of Preventive Medicine, Complejo Hospitalario Universitario Insular Materno Infantil (CHUIMI), Canary Health Service, Las Palmas de Gran Canaria, 35016 Las Palmas, Spain

13 Spanish Nutrition Foundation (FEN), 28010 Madrid, Spain

* Correspondence: gvarela@ceu.es; Tel.: +34-91-372-47-26 or +34-91-447-07-59

Received: 30 August 2019; Accepted: 16 October 2019; Published: 5 November 2019

check for updates

\begin{abstract}
Dietary habits amongst the Spanish population are currently a relevant cause for concern, as macronutrient profiles and micronutrient intakes seem to be inadequate and globally moving away from the traditional Mediterranean dietary pattern. However, recent food consumption patterns have not been fully assessed. In the present study, our aim was therefore to describe the current food consumption from the "anthropometric data, macronutrients and micronutrients intake, practice of physical activity, socioeconomic data and lifestyles in Spain" (ANIBES) study population by assessing data defined by age and gender. The ANIBES study is a cross-sectional study of a nationally representative sample of the Spanish population. A three-day dietary record was used to obtain information about food and beverage consumption. The sample comprised 2009 individuals aged 9-75 years, plus a boost sample for the youngest age groups (9-12, 13-17, and 18-24 years, $n=200$ per age group). The most consumed food group across all age segments were non-alcoholic beverages followed by milk and dairy products and vegetables. Consumption of cereals and derivatives, milk and dairy products, sugars and sweets, and ready-to-eat meals by children was significantly higher than those by the adult and older adult populations $(p \leq 0.05)$. Conversely, intakes of vegetables, fruits, and fish and shellfish were significantly higher in adults and
\end{abstract}


older adults $(p \leq 0.05)$. In order to comply with recommendations, adherence to the Mediterranean dietary patterns should be strengthened, especially amongst younger population groups. Therefore, substantial nutritional interventions may be targeted to improve the Spanish population's dietary patterns nowadays.

Keywords: food group; food subgroup; consumption; intake; dietary pattern; ANIBES study; Spanish population

\section{Introduction}

Food habits and consumption in Spain have experienced a significant change in recent years, leading to a less varied food choice and a tendency towards an increasingly "westernized" diet [1-4]. Namely, the contribution of food and beverage groups to the daily energy intake of the Spanish population from the ANIBES study show that a higher percentage is supplied by cereals and derivatives $(27.4 \%)$, with meat and meat products and oils and fats being second $(15.2 \%)$ and third $(12.3 \%)$, respectively [5]. Therefore, the Spanish diet is characterized by a high content of proteins (derived from fatty, domesticated, and processed meats), saturated fats, refined grains, and sugars. However, the major food groups and subgroups that encompass our dietary patterns remain unassessed.

The current dietary patterns from the Spanish population are moving away from the traditional Mediterranean diet and comprise a higher intake of animal instead of vegetal products such as legumes, nuts, fruits, and vegetables. This concern has already been expressed by other authors, as a high proportion of Spanish young children and adolescents show an even greater distance from the balanced patterns from their adult and elder counterparts [6,7]; potentially, our future generations may not enjoy the health and cultural well-known benefits of the Mediterranean diet $[8,9]$.

At present, it is widely recognized that a suboptimal diet is a major preventable risk factor for non-communicable diseases (NCDs), which in turn, are the leading cause of mortality and disability worldwide [10]. It is estimated that 11 million (95\% uncertainty interval (UI), 10-12) deaths worldwide in 2017 can be attributed to dietary risk factors amongst adults aged 25 years or older [10]. The authors emphasized that intakes of specific foods, within the context of a global healthy diet, instead of macronutrients or micronutrients, might be most relevant for NCD risk [11]. Therefore, the assessment of the consumption of major food groups and subgroups becomes a relevant task.

To date, the study of macro- and micronutrient intakes from the Spanish population has been improved and updated. However, their current food patterns have not been assessed in depth. In the past, most studies analyzed data from the Household Consumption Surveys by the Spanish Ministry of Agriculture, Fisheries and Food, but these might not accurately reflect actual individual population intakes or estimate age and gender differences. Hence, in the present study, our objectives were to analyze and describe the current food patterns from the Spanish ANIBES study population. Consequently, we aimed to assess food group and subgroup consumption defined by age and gender groups of the participants in the ANIBES study as a representative sample of the Spanish population.

\section{Materials and Methods}

The complete design, protocol and methodology of the ANIBES study ("anthropometric data, macronutrients and micronutrients intake, practice of physical activity, socioeconomic data, and lifestyles in Spain") have been described in detail elsewhere [5,12].

\subsection{Sample}

The ANIBES study is a cross-sectional study conducted using stratified multistage sampling. The fieldwork was performed at 128 sampling points across Spain. The design of the study aimed to define a sample size that was representative of all individuals living in Spain, aged 9-75 years, 
and living in municipalities of at least 2000 inhabitants. The initial potential sample consisted of 2634 individuals, and the final sample comprised 2009 individuals (1013 men, 50.4\%; 996 women, $49.6 \%)$. In addition, for the youngest age groups (9-12, 13-17, and 18-24 years), a "boost sample" was included to provide at least $n=200$ per age group (error $\pm 6.9 \%$ ). Therefore, the random sample plus boost comprised 2285 participants. The sample quotas according to the following variables were age groups (9-12, 13-17, 18-64, and 65-75 years) and gender (male/female). The fieldwork for the ANIBES study was conducted from mid-September 2013 to mid-November 2013, and two previous pilot studies were performed. The final protocol was approved by the Ethical Committee for Clinical Research of the Region of Madrid, Spain. The study was coded as "FEN 2013" and approved on 31 May 2013 [5].

\subsection{Food and Beverage Records and Adequacy of Intakes}

Study participants were provided with a tablet device (Samsung Galaxy Tab 2 7.0, Samsung Electronics, Suwon, Korea) and trained in recording information by taking photos of all food and drinks consumed during the 3 days of the study, both at and outside the home. Different days of the week would be equally represented as each 3 day cycle included two working days and one weekend day. Photos had to be taken before and after each eating and drinking occasion, in order to record the actual intake. Additionally, a brief description of the meals, recipes, food brands, and other relevant information were recorded using the tablet. Participants who declared that they were unable to use the device were offered other options, such as using a digital camera, handwritten records, and/or telephone interviews. A total of $79 \%$ of the sample used a tablet, $12 \%$ a digital camera, and $9 \%$ opted for a telephone interview. Participants provided detailed information regarding each eating and drinking occasion including not only what and how much was eaten, but also where and with whom they shared their meals, and if they were watching television and/or sitting at a table. In addition, they were asked to declare if their intake was representative for that specific day (e.g., in case of a medical condition such as gastroenteritis that might compromise dietary habits) and to record any dietary supplement taken. The survey also included a series of questions about participants' customary eating habits (e.g., the type of milk usually consumed) to facilitate further coding. Food records were returned from the field in real time to be coded by trained personal who were supervised by dieticians.

\subsection{Statistical Analysis}

Results are expressed as means \pm standard deviation (SD) per group. Comparisons among groups were performed using a Student's $t$-test for independent samples to evaluate differences by sex within the whole population and within each age group. One-way analysis of variance (ANOVA) tests with Bonferroni correction for multiple comparisons was used to calculate differences among each age group. Differences were considered significant at $p<0.05$. Data analysis was performed with the SPSS 24.0 software package (IBM Corp., Armonk, NY, USA).

\section{Results}

The statistical description of the ANIBES population sample is included in Table 1. Data analysis on food consumption allows estimation of the average Spanish daily menu and the associated distribution of the different food groups, as shown in Table 2. Non-alcoholic beverage consumption was quantitatively the most important in the present Spanish diet followed by milk and dairy products, vegetables, fruits, grains, and meat and meat products. The most relevant consumption, within the group of non-alcoholic beverages, was associated with bottled water followed by sugary soft drinks (Table 2), whereas within the milk and dairy products, the highest consumption was recorded for liquid milk. In addition, we observed that men consumed significantly higher quantities of cereals and derivatives, meat and meat products, eggs, ready-to eat meals, sauces and condiments, and alcoholic beverages than women. However, females had higher intakes of fruits $(p<0.05)$ when compared to males (Table 2). 
Table 1. Statistical description of the(ANIBES) "anthropometric data, macronutrients and micronutrients intake, practice of physical activity, socioeconomic data, and lifestyles in Spain" study population sample (Modified from Nissensohn et al. [13]).

\begin{tabular}{|c|c|c|c|c|c|c|c|}
\hline & & Total & $\%$ & Male & $\%$ & Female & $\%$ \\
\hline & & 2007 & 100 & 1011 & 50.4 & 996 & 49.6 \\
\hline \multirow{4}{*}{ Age Group } & Children (9-12 years) & 100 & 5.0 & 62 & 6.1 & 38 & 3.8 \\
\hline & Adolescents (13-17 years) & 123 & 6.1 & 84 & 8.3 & 39 & 3.9 \\
\hline & Adults (18-64 years) & 1587 & 79.1 & 772 & 76.4 & 815 & 81.9 \\
\hline & Older adults (65-75 years) & 197 & 9.8 & 93 & 9.2 & 104 & 10.4 \\
\hline \multirow{3}{*}{ Level of education } & Primary or less & 743 & 37 & 378 & 37.4 & 365 & 36.6 \\
\hline & Secondary & 858 & 42.8 & 434 & 42.9 & 424 & 42.6 \\
\hline & Tertiary or University & 406 & 20.2 & 199 & 19.7 & 207 & 20.8 \\
\hline \multirow{5}{*}{ Economical level } & $1000 €$ or less & 397 & 19.8 & 191 & 18.9 & 206 & 20.7 \\
\hline & From 1000 to $2000 €$ & 795 & 39.6 & 393 & 38.9 & 402 & 40.4 \\
\hline & Over $2000 €$ & 320 & 15.9 & 163 & 16.1 & 157 & 15.8 \\
\hline & No income & 7 & 0.3 & 4 & 0.4 & 3 & 0.3 \\
\hline & No answer & 488 & 24.3 & 260 & 25.7 & 228 & 22.9 \\
\hline \multirow{2}{*}{ Geographical distribution } & Northwest & 152 & 7.6 & 77 & 7.6 & 75 & 7.5 \\
\hline & North Central & 161 & 8 & 79 & 7.8 & 82 & 8.2 \\
\hline
\end{tabular}

Similarly, in all age segments of the ANIBES population, the most consumed food group was non-alcoholic beverages (mainly water) followed by milk and dairy products and vegetables. However, in the elderly (65-75 years), the second most consumed group were fruits, followed by dairy products (Table 3). It is important to highlight that the consumption of cereals and derivatives, milk and dairy products, meats and meat products, sugars and sweets, appetizers, ready-to-eat meals, and sauces and condiments were significantly higher in children than the intakes by the adult and older adult populations $(p \leq 0.05)$. In particular, milk and dairy products intake were significantly higher in children compared to adolescents $(p \leq 0.05)$. On the contrary, intakes of vegetables, fruits, oils and fats, fish and shellfish, and alcoholic beverages were significantly higher in adults and the elderly $(p \leq 0.05)$. Similarly, it should be noted that, although the vegetable consumption was higher in adults and older adults when compared to the infant-juvenile population, older adults were the only group with a significantly higher consumption of this food group $(p \leq 0.05)$. However, adults between 18 and 64 years old consumed the highest quantities of drinks $(p \leq 0.05)$; both alcoholic and non-alcoholic (Table 3). 
Table 2. Daily food groups and subgroups intake distribution amongst the Spanish ANIBES study population (9-75 years).

\begin{tabular}{|c|c|c|c|}
\hline Food Groups and Subgroups & Total Population & Men & Women \\
\hline Cereals and derivatives (g/day) & $148.9 \pm 63.8$ & $163.8 \pm 67.9$ & $133.8 \pm 55.4^{* * *}$ \\
\hline Grains and flours (g/day) & $22.5 \pm 26.4$ & $23.8 \pm 28.6$ & $21.2 \pm 23.9 *$ \\
\hline Breakfast cereals and cereal bars (g/day) & $4.7 \pm 12.9$ & $5.0 \pm 14.9$ & $4.4 \pm 10.5$ \\
\hline Bread (g/day) & $75.0 \pm 45.2$ & $84.9 \pm 49.6$ & $64.9 \pm 37.8^{* * *}$ \\
\hline $\operatorname{Pasta}(\mathrm{g} /$ day $)$ & $16.9 \pm 20.9$ & $19.2 \pm 23.1$ & $14.6 \pm 18.1^{* * *}$ \\
\hline Bakery and pastry (g/day) & $29.8 \pm 33.9$ & $30.9 \pm 35.7$ & $28.8 \pm 31.9$ \\
\hline Vegetables (g/day) & $177.8 \pm 112.9$ & $175.3 \pm 116.0$ & $180.3 \pm 109.6$ \\
\hline Fruits(g/day) & $158.0 \pm 175.4$ & $149.6 \pm 173.9$ & $166.5 \pm 176.6^{*}$ \\
\hline Oils and fats (g/day) & $24.5 \pm 11.2$ & $24.8 \pm 11.5$ & $24.3 \pm 10.8$ \\
\hline Olive oil (g/day) & $17.5 \pm 9.4$ & $17.8 \pm 9.8$ & $17.1 \pm 9.0$ \\
\hline Other oils (g/day) & $3.6 \pm 5.1$ & $3.9 \pm 5.6$ & $3.3 \pm 4.6^{* *}$ \\
\hline Butter. margarine and shortening (g/day) & $3.5 \pm 7.0$ & $3.1 \pm 7.0$ & $3.9 \pm 7.1^{* *}$ \\
\hline Milk and dairy products (g/day) & $257.2 \pm 159.5$ & $258.4 \pm 170.5$ & $256.1 \pm 146.6$ \\
\hline Milks (g/day) & $173.4 \pm 132.8$ & $171.1 \pm 139.0$ & $175.8 \pm 126.3$ \\
\hline Cheeses (g/day) & $18.0 \pm 23.0$ & $18.4 \pm 66.7$ & $17.6 \pm 60.7$ \\
\hline Yogurt and fermented milk (g/day) & $46.5 \pm 63.7$ & $46.2 \pm 23.9$ & $46.8 \pm 19.7$ \\
\hline Other dairy products (g/day) & $19.4 \pm 44.1$ & $22.8 \pm 50.6$ & $16.0 \pm 35.8^{* * *}$ \\
\hline Fish and shellfish (g/day) & $62.3 \pm 69.9$ & $60.8 \pm 68.2$ & $63.9 \pm 71.6$ \\
\hline Meat and meat products (g/day) & $146.0 \pm 82.8$ & $165.4 \pm 87.8$ & $126.3 \pm 72.2 * * *$ \\
\hline Meat (g/day) & $103.9 \pm 73.8$ & $117.4 \pm 78.8$ & $90.3 \pm 65.6^{* * *}$ \\
\hline Viscera and spoils (g/day) & $40.5 \pm 36.4$ & $46.1 \pm 9.6$ & $34.7 \pm 7.6$ \\
\hline Sausages and other meat products (g/day) & $1.6 \pm 8.7$ & $1.9 \pm 39.6$ & $1.3 \pm 31.8^{* * *}$ \\
\hline Eggs (g/day) & $29.3 \pm 29.4$ & $32.8 \pm 32.9$ & $25.7 \pm 25.0^{* * *}$ \\
\hline Pulses (g/day) & $13.5 \pm 17.8$ & $14.1 \pm 18.8$ & $12.8 \pm 16.8$ \\
\hline Sugar and sweets (g/day) & $15.9 \pm 16.0$ & $15.4 \pm 16.1$ & $16.4 \pm 16.0$ \\
\hline Sugar (g/day) & $6.2 \pm 8.4$ & $6.0 \pm 8.2$ & $6.5 \pm 8.5$ \\
\hline Chocolates (g/day) & $6.8 \pm 11.4$ & $7.2 \pm 11.9$ & $6.4 \pm 10.9$ \\
\hline Jams and other (g/day) & $2.2 \pm 6.4$ & $1.7 \pm 5.7$ & $2.7 \pm 7.1^{* * *}$ \\
\hline Other sweets (g/day) & $0.7 \pm 3.7$ & $0.5 \pm 3.2$ & $0.9 \pm 4.2$ \\
\hline Appetizers (g/day) & $5.5 \pm 12.3$ & $5.9 \pm 12.6$ & $5.1 \pm 1.9$ \\
\hline Ready-to-eat-meals (g/day) & $70.2 \pm 77.6$ & $79.4 \pm 86.2$ & $60.9 \pm 66.5^{* * *}$ \\
\hline Sauces and condiments (g/day) & $12.9 \pm 14.5$ & $14.4 \pm 15.3$ & $11.4 \pm 13.5^{* * *}$ \\
\hline Non-alcoholic Beverages (g/day) & $850.8 \pm 521.3$ & $842.1 \pm 540.6$ & $859.7 \pm 501.1$ \\
\hline Water (g/day) & $569.7 \pm 502.3$ & $557.7 \pm 517.9$ & $581.9 \pm 485.8$ \\
\hline Coffee and infusions (g/day) & $86.2 \pm 117.9$ & $75.0 \pm 109.2$ & $97.6 \pm 125.2 * * *$ \\
\hline Sugary soft drinks (g/day) & $88.2 \pm 151.3$ & $103.2 \pm 170.9$ & $73.0 \pm 126.5^{* * *}$ \\
\hline Non-sweetened soft drinks (g/day) & $35.4 \pm 108.8$ & $29.2 \pm 91.9$ & $41.6 \pm 123.3 * *$ \\
\hline Sports Drinks (g/day) & $4.9 \pm 33.9$ & $6.8 \pm 41.3$ & $2.9 \pm 24.1^{* *}$ \\
\hline Juices and nectars (g/day) & $50.6 \pm 94.4$ & $56.3 \pm 104.0$ & $44.9 \pm 83.2 * *$ \\
\hline Other drinks (non-alcoholic) (g/day) & $14.2 \pm 55.6$ & $11.5 \pm 55.0$ & $16.9 \pm 56.1 *$ \\
\hline Energy drinks (g/day) & $1.7 \pm 20.6$ & $2.4 \pm 26.8$ & $1.0 \pm 11.2$ \\
\hline Alcoholic Beverages (g/day) & $99.2 \pm 195.8$ & $135.4 \pm 238.0$ & $62.4 \pm 130.8^{* * *}$ \\
\hline High alcohol content (g/day) & $2.2 \pm 13.5$ & $2.8 \pm 15.1$ & $1.5 \pm 11.6^{* *}$ \\
\hline Low alcohol content (g/day) & $97.0 \pm 192.9$ & $132.6 \pm 234.0$ & $60.9 \pm 129.5^{* * *}$ \\
\hline Supplements and meal replacements (g/day) & $0.4 \pm 4.6$ & $0.5 \pm 5.1$ & $0.3 \pm 4.0$ \\
\hline
\end{tabular}

Data reported as means \pm standard deviation (SD) per group. ${ }^{*} p<0.05$ difference male (Student's $t$-test); ${ }^{* *} p<0.01$ difference male (Student's t-test); ${ }^{* * *} p<0.001$ difference male (Student's $t$-test). 
Table 3. Total daily food groups and subgroups intake by age amongst the Spanish ANIBES study population.

\begin{tabular}{|c|c|c|c|c|}
\hline Food Groups and Subgroups & $\begin{array}{l}\text { Children } \\
\text { (9-12 years) }\end{array}$ & $\begin{array}{l}\text { Adolescents } \\
\text { (13-17 years) }\end{array}$ & $\begin{array}{c}\text { Adults } \\
\text { (18-64 years) }\end{array}$ & $\begin{array}{l}\text { Older Adults } \\
\text { (65-75 years) }\end{array}$ \\
\hline Cereals and derivatives (g/day) & $168.4 \pm 58.6^{\mathrm{a}, \mathrm{b}}$ & $180.8 \pm 71.9^{\mathrm{a}}$ & $148.8 \pm 64.4^{\mathrm{b}}$ & $126.3 \pm 53.2^{c}$ \\
\hline Grains and flours (g/day) & $21.1 \pm 23.0$ & $25.1 \pm 27.1$ & $22.8 \pm 26.9$ & $17.6 \pm 22.0$ \\
\hline Breakfast cereals and cereal bars (g/day) & $7.8 \pm 15.7$ & $9.7 \pm 18.5$ & $4.4 \pm 12.1$ & $3.0 \pm 9.7$ \\
\hline Bread (g/day) & $78.4 \pm 44.7$ & $83.7 \pm 52.5$ & $75.0 \pm 45.4$ & $70.9 \pm 44.3$ \\
\hline Pasta(g/day) & $20.3 \pm 17.5$ & $22.8 \pm 25.1$ & $17.2 \pm 21.3$ & $10.0 \pm 14.0$ \\
\hline Bakery and pastry (g/day) & $40.8 \pm 32.4$ & $39.5 \pm 39.9$ & $29.4 \pm 33.7$ & $24.9 \pm 29.6$ \\
\hline Vegetables (g/day) & $124.8 \pm 92.9^{\mathrm{d}}$ & $122.2 \pm 85.0^{\mathrm{d}}$ & $181.1 \pm 115.3^{\mathrm{e}}$ & $210.3 \pm 110.4^{\mathrm{f}}$ \\
\hline Fruits $(\mathrm{g} / \mathrm{d})$ & $104.9 \pm 104.1^{g}$ & $87.0 \pm 114.7^{g}$ & $151.5 \pm 170.4^{\mathrm{h}}$ & $280.8 \pm 222.0^{\mathrm{i}}$ \\
\hline Oils and fats (g/day) & $23.0 \pm 9.5^{\mathrm{j}, \mathrm{k}}$ & $22.2 \pm 12.3^{j}$ & $24.6 \pm 11.1^{\mathrm{j}, \mathrm{k}}$ & $26.8 \pm 13.2^{\mathrm{k}}$ \\
\hline Olive oil (g/day) & $15.1 \pm 7.9$ & $14.3 \pm 9.2$ & $17.4 \pm 9.3$ & $21.0 \pm 9.9$ \\
\hline Other oils (g/day) & $4.4 \pm 5.2$ & $4.5 \pm 7.1$ & $3.7 \pm 5.2$ & $1.4 \pm 2.9$ \\
\hline Butter, margarine, and shortening ( $\mathrm{g} /$ day) & $3.5 \pm 6.2$ & $3.5 \pm 7.9$ & $3.4 \pm 6.6$ & $4.3 \pm 10.6$ \\
\hline Milk and dairy products (g/day) & $360.1 \pm 149.6^{1}$ & $307.0 \pm 188.3^{\mathrm{m}}$ & $247.6 \pm 154.8^{\mathrm{n}}$ & $260.4 \pm 159.7^{n}$ \\
\hline Milks (g/day) & $232.7 \pm 132.0$ & $216.5 \pm 134.9$ & $165.8 \pm 129.9$ & $184.6 \pm 140.3$ \\
\hline Cheeses (g/day) & $18.5 \pm 64.7$ & $18.7 \pm 64.6$ & $18.5 \pm 62.2$ & $12.7 \pm 70.7$ \\
\hline Yogurt and fermented milk (g/day) & $58.3 \pm 16.2$ & $39.1 \pm 18.2$ & $44.9 \pm 22.9$ & $54.9 \pm 16.7$ \\
\hline Other dairy products (g/day) & $50.6 \pm 76.7$ & $32.8 \pm 61.6$ & $18.4 \pm 40.5$ & $8.2 \pm 20.8$ \\
\hline Fish and shellfish (g/day) & $45.4 \pm 54.6^{\mathrm{m}}$ & $40.2 \pm 57.9^{\mathrm{m}}$ & $62.6 \pm 70.4^{\circ}$ & $81.1 \pm 72.5^{\mathrm{P}}$ \\
\hline Meat and meat products (g/day) & $148.4 \pm 69.5^{\mathrm{q}}$ & $158.8 \pm 71.4^{\mathrm{q}}$ & $148.4 \pm 84.4^{\mathrm{q}}$ & $117.0 \pm 70.0^{\mathrm{r}}$ \\
\hline Meat (g/day) & $97.1 \pm 61.8$ & $109.4 \pm 69.8$ & $106.2 \pm 75.6$ & $85.9 \pm 61.3$ \\
\hline Viscera and spoils (g/day) & $51.0 \pm 3.2$ & $48.9 \pm 5.1$ & $40.7 \pm 8.3$ & $27.9 \pm 13.2$ \\
\hline Sausages and other meat products (g/day) & $0.3 \pm 39.0$ & $0.5 \pm 36.9$ & $1.5 \pm 36.4$ & $3.2 \pm 28.9$ \\
\hline $\operatorname{Eggs}(\mathrm{g} /$ day $)$ & $27.0 \pm 24.4$ & $31.3 \pm 34.1$ & $29.3 \pm 29.5$ & $32.4 \pm 29.2$ \\
\hline Pulses (g/day) & $12.2 \pm 14.3$ & $12.1 \pm 16.2$ & $13.4 \pm 18.0$ & $15.2 \pm 18.6$ \\
\hline Sugar and sweets (g/day) & $24.6 \pm 19.9^{\mathrm{s}}$ & $21.9 \pm 18.7^{\mathrm{s}}$ & $15.6 \pm 15.5^{\mathrm{t}}$ & $12.3 \pm 13.7^{\mathrm{u}}$ \\
\hline Sugar (g/day) & $2.6 \pm 5.2$ & $4.0 \pm 7.4$ & $6.7 \pm 8.7$ & $5.4 \pm 7.6$ \\
\hline Chocolates (g/day) & $19.1 \pm 17.3$ & $15.5 \pm 15.1$ & $6.1 \pm 10.1$ & $2.0 \pm 5.3$ \\
\hline Jams and other (g/day) & $1.3 \pm 4.0$ & $1.1 \pm 4.0$ & $2.1 \pm 6.2$ & $4.7 \pm 10.2$ \\
\hline Other sweets (g/day) & $1.5 \pm 5.5$ & $1.3 \pm 5.6$ & $0.7 \pm 3.7$ & $0.2 \pm 0.7$ \\
\hline Appetizers (g/day) & $5.1 \pm 10.1^{\mathrm{v}, \mathrm{w}}$ & $5.7 \pm 12.5^{\mathrm{v}, \mathrm{w}}$ & $5.8 \pm 12.7^{\mathrm{v}}$ & $2.9 \pm 9.0^{\mathrm{w}}$ \\
\hline Ready-to-eat meals (g/day) & $83.0 \pm 75.3^{x}$ & $95.8 \pm 93.4^{x}$ & $67.5 \pm 75.7^{y}$ & $60.3 \pm 68.3^{y}$ \\
\hline Sauces and condiments (g/day) & $15.9 \pm 17.0^{\mathrm{z}}$ & $15.4 \pm 15.0^{\mathrm{z}}$ & $13.3 \pm 14.8^{\mathrm{z}}$ & $6.8 \pm 10.2^{\alpha}$ \\
\hline Non-alcoholic beverages (g/day) & $707.8 \pm 408.8^{\beta}$ & $699.4 \pm 381.3^{\beta}$ & $885.1 \pm 535.5^{\Omega}$ & $700.5 \pm 435.2^{\beta}$ \\
\hline Water (g/day) & $481.4 \pm 387.3$ & $407.1 \pm 358.4$ & $591.3 \pm 519.8$ & $496.9 \pm 414.7$ \\
\hline Coffee and infusions (g/day) & $4.1 \pm 18.2$ & $11.2 \pm 37.9$ & $93.9 \pm 115.1$ & $112.2 \pm 159.1$ \\
\hline Sugar soft drinks (g/day) & $84.4 \pm 139.0$ & $150.6 \pm 190.0$ & $89.0 \pm 149.8$ & $23.8 \pm 63.6$ \\
\hline Non-sweetened soft drinks (g/day) & $15.1 \pm 46.8$ & $20.5 \pm 76.2$ & $41.0 \pm 118.6$ & $13.1 \pm 50.8$ \\
\hline Sports drinks (g/day) & $9.5 \pm 51.0$ & $2.0 \pm 16.5$ & $6.0 \pm 40.6$ & $1.0 \pm 10.4$ \\
\hline Juices and nectars (g/day) & $111.7 \pm 137.8$ & $99.7 \pm 147.7$ & $46.6 \pm 86.4$ & $33.0 \pm 78.1$ \\
\hline Other drinks (non-alcoholic) (g/day) & $1.2 \pm 17.1$ & $4.4 \pm 28.9$ & $15.9 \pm 58.8$ & $20.5 \pm 69.4$ \\
\hline Energy drinks (g/day) & $0.5 \pm 7.5$ & $3.9 \pm 29.1$ & $1.5 \pm 20.1$ & $0.0 \pm 0.0$ \\
\hline Alcoholic beverages (g/day) & $0.0 \pm 0.0^{\pi}$ & $1.8 \pm 16.0^{\pi}$ & $113.4 \pm 209.1^{\mu}$ & $96.4 \pm 156.3^{\mu}$ \\
\hline High alcohol content beverage (g/day) & $0.0 \pm 0.0$ & $0.0 \pm 0.0$ & $2.5 \pm 14.3$ & $2.1 \pm 14.5$ \\
\hline Low alcohol content beverage (g/day) & $0.0 \pm 0.0$ & $1.8 \pm 16.0$ & $110.8 \pm 206.1$ & $94.3 \pm 153.7$ \\
\hline Supplements and meal replacements (g/day) & $0.0 \pm 0.2$ & $0.1 \pm 1.5$ & $0.4 \pm 5.0$ & $0.6 \pm 5.7$ \\
\hline
\end{tabular}

Data reported as means \pm standard deviation (SD) per group. Different superscript lowercase letters indicate statistical significance in each row for food groups ( $p \leq 0.05$; Bonferroni test).

Male children and adolescents consumed higher levels of meats and processed meats, milk and derivatives, sugar and sweets, ready-to-eat meals, and sauces and condiments than adult groups $(p<0.05)$ (Table 4). In addition, children, adolescents, and adults showed a higher meat and derivative consumption than older adults $(p<0.05)$. Conversely, adults and elderly male populations presented a higher intake of vegetables, fruits, fats and oils, and fish and shellfish than children and adolescents $(p<0.05)$. Lastly, it is noteworthy that adults had a higher consumption of beverages, non-alcoholic as well as alcoholic $(p<0.05)$. 
Table 4. Total daily food groups and subgroups intake by age in the male Spanish ANIBES study population.

\begin{tabular}{|c|c|c|c|c|}
\hline \multirow{2}{*}{ Food Groups and Subgroups } & Children & Adolescents & Adults & Older Adults \\
\hline & (9-12 years) & (13-17 years) & (18-64 years) & (65-75 years) \\
\hline Cereals and derivatives (g/day) & $169.9 \pm 60.2^{a}$ & $192.6 \pm 74.6^{b}$ & $163.7 \pm 68.5^{\mathrm{a}}$ & $137.2 \pm 59.0^{c}$ \\
\hline Grains and flours (g/day) & $20.9 \pm 20.3$ & $25.7 \pm 28.3$ & $24.2 \pm 29.5$ & $20.1 \pm 25.2$ \\
\hline Breakfast cereals and cereal bars (g/day) & $8.2 \pm 17.9$ & $11.4 \pm 21.2$ & $4.3 \pm 13.5$ & $3.1 \pm 8.9$ \\
\hline Bread (g/day) & $80.2 \pm 45.9$ & $88.9 \pm 54.6$ & $85.5 \pm 50.1$ & $79.1 \pm 47.7$ \\
\hline Pasta $(\mathrm{g} /$ day $)$ & $20.3 \pm 16.8$ & $24.9 \pm 26.1$ & $19.9 \pm 23.9$ & $9.2 \pm 13.0$ \\
\hline Bakery and pastry (g/day) & $40.4 \pm 32.8$ & $41.8 \pm 44.8$ & $29.8 \pm 34.9$ & $25.8 \pm 32.6$ \\
\hline Vegetables (g/day) & $120.6 \pm 78.0^{\mathrm{d}}$ & $123.7 \pm 90.0^{\mathrm{d}}$ & $181.0 \pm 118.7^{\mathrm{e}}$ & $214.7 \pm 108.0^{f}$ \\
\hline Fruits(g/day) & $96.7 \pm 96.2 \mathrm{~g}$ & $82.0 \pm 116.9 \mathrm{~g}$ & $147.5 \pm 167.8^{h}$ & $279.0 \pm 244.9^{i}$ \\
\hline Oils and fats (g/day) & $23.1 \pm 9.9^{j}$ & $23.0 \pm 12.7^{\mathrm{j}}$ & $24.8 \pm 11.3^{\mathrm{j}, \mathrm{k}}$ & $27.7 \pm 13.2^{\mathrm{k}}$ \\
\hline Olive oil (g/day) & $15.3 \pm 8.3$ & $15.1 \pm 10.0$ & $17.8 \pm 9.8$ & $22.4 \pm 9.8$ \\
\hline Other oils (g/day) & $4.2 \pm 4.8$ & $4.4 \pm 6.8$ & $4.1 \pm 5.6$ & $1.6 \pm 3.3$ \\
\hline Butter, margarine and shortening (g/day) & $3.6 \pm 6.5$ & $3.5 \pm 8.5$ & $2.9 \pm 6.1$ & $3.7 \pm 10.8$ \\
\hline Milk and dairy products (g/day) & $384.2 \pm 148.7^{1}$ & $331.6 \pm 200.0^{1}$ & $246.5 \pm 167.0^{\mathrm{m}}$ & $242.3 \pm 159.6^{\mathrm{m}}$ \\
\hline Milks (g/day) & $252.2 \pm 137.0$ & $241.0 \pm 177.2$ & $160.2 \pm 136.5$ & $176.9 \pm 145.1$ \\
\hline Cheeses (g/day) & $20.0 \pm 23.3$ & $18.3 \pm 22.7$ & $19.1 \pm 26.1$ & $10.7 \pm 14.8$ \\
\hline Yogurt and fermented milk (g/day) & $57.9 \pm 62.0$ & $36.6 \pm 54.8$ & $46.2 \pm 68.3$ & $47.7 \pm 67.5$ \\
\hline Other dairy products (g/day) & $54.1 \pm 80.0$ & $35.7 \pm 67.8$ & $20.9 \pm 45.3$ & $7.0 \pm 17.9$ \\
\hline Fish and shellfish (g/day) & $45.7 \pm 59.4^{\mathrm{n}}$ & $40.2 \pm 55.3^{n}$ & $61.4 \pm 68.6^{\circ}$ & $90.3 \pm 76.7 \mathrm{p}$ \\
\hline Meat and meat products (g/day) & $159.3 \pm 73.0^{\mathrm{q}, \mathrm{r}}$ & $171.4 \pm 66.0^{\mathrm{r}}$ & $169.2 \pm 73.6^{\mathrm{r}}$ & $132.5 \pm 76.0 \mathrm{q}$ \\
\hline Meat (g/day) & $107.7 \pm 64.5$ & $120.2 \pm 70.1$ & $120.7 \pm 81.2$ & $95.5 \pm 66.4$ \\
\hline Sausages and other meat products (g/day) & $51.6 \pm 1.5$ & $50.9 \pm 2.8$ & $46.6 \pm 9.7$ & $33.1 \pm 13.6$ \\
\hline Viscera and spoils (g/day) & $0.1 \pm 1.5$ & $0.2 \pm 2.8$ & $1.9 \pm 9.7$ & $3.9 \pm 13.6$ \\
\hline Eggs (g/day) & $27.7 \pm 25.6$ & $34.7 \pm 36.1$ & $33.2 \pm 33.4$ & $34.5 \pm 29.5$ \\
\hline Pulses (g/day) & $11.0 \pm 14.8$ & $12.0 \pm 15.3$ & $14.3 \pm 19.2$ & $17.3 \pm 20.0$ \\
\hline Sugar and sweets (g/day) & $24.4 \pm 21.0^{\mathrm{s}}$ & $20.8 \pm 16.4^{\mathrm{s}}$ & $14.8 \pm 15.5^{\mathrm{t}}$ & $12.7 \pm 14.0^{\mathrm{t}}$ \\
\hline Sugar (g/day) & $2.6 \pm 5.1$ & $4.3 \pm 8.1$ & $6.6 \pm 8.8$ & $5.6 \pm 7.4$ \\
\hline Chocolates (g/day) & $19.2 \pm 17.5$ & $14.6 \pm 13.8$ & $6.2 \pm 10.6$ & $3.0 \pm 6.4$ \\
\hline Jams and other (g/day) & $1.1 \pm 3.6$ & $1.0 \pm 3.7$ & $1.6 \pm 5.4$ & $4.0 \pm 9.5$ \\
\hline Other sweets (g/day) & $1.4 \pm 6.4$ & $0.9 \pm 3.5$ & $0.5 \pm 2.9$ & $0.2 \pm 0.7$ \\
\hline Appetizers (g/day) & $5.0 \pm 9.8$ & $5.4 \pm 12.4$ & $6.4 \pm 13.3$ & $3.3 \pm 8.4$ \\
\hline Ready-to-eat meals (g/day) & $88.9 \pm 72.9^{\mathrm{u}, \mathrm{v}}$ & $107.5 \pm 101.2^{\mathrm{u}}$ & $74.1 \pm 84.1^{\mathrm{v}}$ & $76.3 \pm 74.5^{\mathrm{v}}$ \\
\hline Sauces and condiments (g/day) & $14.4 \pm 12.9^{\mathrm{x}}$ & $16.0 \pm 13.9^{x}$ & $15.0 \pm 15.9^{\mathrm{x}}$ & $7.5 \pm 11.6^{y}$ \\
\hline Non-alcoholic beverages (g/day) & $728.4 \pm 435.2^{z}$ & $714.0 \pm 418.4^{\mathrm{z}}$ & $884.2 \pm 561.4^{\alpha}$ & $640.8 \pm 429.4^{Z}$ \\
\hline Water (g/day) & $487.6 \pm 402.6$ & $416.8 \pm 387.3$ & $584.1 \pm 541.8$ & $452.7 \pm 403.1$ \\
\hline Coffee and infusions (g/day) & $2.6 \pm 13.0$ & $12.8 \pm 43.4$ & $86.0 \pm 114.1$ & $88.7 \pm 106.7$ \\
\hline Sugar soft drinks (g/day) & $99.1 \pm 158.2$ & $158.6 \pm 215.3$ & $102.5 \pm 167.2$ & $24.6 \pm 63.7$ \\
\hline Non-sweetened soft drinks (g/day) & $12.0 \pm 35.3$ & $21.5 \pm 74.4$ & $34.8 \pm 103.5$ & $8.0 \pm 31.2$ \\
\hline Sports drinks (g/day) & $13.0 \pm 61.9$ & $1.3 \pm 11.0$ & $8.8 \pm 51.9$ & $1.3 \pm 13.4$ \\
\hline Juices and nectars (g/day) & $112.0 \pm 146.8$ & $94.8 \pm 137.6$ & $53.3 \pm 100.8$ & $34.3 \pm 79.6$ \\
\hline Other drinks (non-alcoholic) (g/day) & $2.0 \pm 22.3$ & $2.0 \pm 12.4$ & $12.6 \pm 58.0$ & $31.3 \pm 87.0$ \\
\hline Energy drinks (g/day) & $0.0 \pm 0.0$ & $6.1 \pm 35.9$ & $2.1 \pm 26.4$ & $0.0 \pm 0.0$ \\
\hline Alcoholic beverages (g/day) & $0.0 \pm 0.0^{\beta}$ & $1.1 \pm 1.1^{\beta}$ & $159.3 \pm 9.0^{\mu}$ & $143.5 \pm 19.0^{\mu}$ \\
\hline High alcohol content beverage (g/day) & $0.0 \pm 0.0$ & $0.0 \pm 0.0$ & $3.2 \pm 15.6$ & $4.4 \pm 20.8$ \\
\hline Low alcohol content beverage (g/day) & $0.0 \pm 0.0$ & $1.1 \pm 13.2$ & $156.0 \pm 251.0$ & $139.1 \pm 185.6$ \\
\hline Supplements and meal replacements (g/day) & $0.0 \pm 0.3$ & $0.2 \pm 1.9$ & $0.5 \pm 5.7$ & $1.1 \pm 8.2$ \\
\hline
\end{tabular}

Data reported as means \pm standard deviation (SD) per group. Different superscript lowercase letters indicate statistical significance in each row for food groups ( $p \leq 0.05$; Bonferroni test).

Children and adolescent females consumed more cereals and derivatives, meat and meat products, sugars and sweets, prepared meals, and sauces and condiments $(p<0.05)$ (Table 5). Likewise, it should be noted that girls consumed more milk and derivatives than the rest of the other population groups $(p<0.05)$. On the contrary, adult or elderly women had a higher consumption of vegetables, fruits, fish and shellfish and alcoholic beverages $(p<0.05)$. In addition, adult females had the highest consumption of non-alcoholic beverages. 
Table 5. Total daily food groups and subgroups intake by age in the female Spanish ANIBES study population.

\begin{tabular}{|c|c|c|c|c|}
\hline \multirow{2}{*}{ Food Groups and Subgroups } & Children & Adolescents & Adults & Older Adults \\
\hline & (9-12 years) & (13-17 years) & (18-64 years) & (65-75 years) \\
\hline Cereals and derivatives (g/day) & $166.3 \pm 58.5^{\mathrm{a}}$ & $158.9 \pm 63.2^{\mathrm{a}}$ & $134.9 \pm 56.4^{b}$ & $116.2 \pm 45.5^{b}$ \\
\hline Grains and flours (g/day) & $21.4 \pm 26.5$ & $23.8 \pm 24.8$ & $21.5 \pm 24.2$ & $15.3 \pm 18.4$ \\
\hline Breakfast cereals and cereal bars (g/day) & $7.3 \pm 11.9$ & $6.7 \pm 11.6$ & $4.5 \pm 10.6$ & $2.9 \pm 10.4$ \\
\hline Bread (g/day) & $75.8 \pm 43.2$ & $73.9 \pm 47.2$ & $65.1 \pm 38.1$ & $63.3 \pm 39.6$ \\
\hline $\operatorname{Pasta}(\mathrm{g} /$ day $)$ & $20.4 \pm 18.5$ & $19.1 \pm 22.7$ & $14.7 \pm 18.2$ & $10.7 \pm 14.9$ \\
\hline Bakery and pastry (g/day) & $41.4 \pm 31.9$ & $35.4 \pm 28.5$ & $29.0 \pm 32.6$ & $24.0 \pm 29.2$ \\
\hline Vegetables (g/day) & $130.7 \pm 111.2^{\mathrm{c}}$ & $119.5 \pm 75.4^{\mathrm{c}}$ & $181.3 \pm 112.2^{\mathrm{d}}$ & $206.3 \pm 113.0^{d}$ \\
\hline Fruits (g/day) & $117.0 \pm 114.1^{\mathrm{e}, \mathrm{f}}$ & $96.2 \pm 110.5^{\mathrm{e}}$ & $155.3 \pm 172.7^{\mathrm{f}}$ & $282.5 \pm 199.6^{g}$ \\
\hline Oils and fats (g/day) & $22.9 \pm 8.8^{\mathrm{h}, \mathrm{i}}$ & $20.8 \pm 11.6^{\mathrm{h}}$ & $24.5 \pm 10.9^{\mathrm{i}}$ & $25.9 \pm 13.3^{i}$ \\
\hline Olive oil (g/day) & $14.8 \pm 7.2$ & $12.7 \pm 7.2$ & $17.1 \pm 8.9$ & $19.7 \pm 9.8$ \\
\hline Other oils (g/day) & $4.7 \pm 5.7$ & $4.6 \pm 7.6$ & $3.4 \pm 4.6$ & $1.3 \pm 2.6$ \\
\hline Butter, margarine and shortening (g/day) & $3.4 \pm 5.7$ & $3.5 \pm 6.7$ & $3.9 \pm 7.0$ & $4.9 \pm 10.5$ \\
\hline Milk and dairy products (g/day) & $325.1 \pm 151.0^{j}$ & $261.4 \pm 154.9^{\mathrm{k}}$ & $248.6 \pm 142.8^{\mathrm{k}}$ & $277.2 \pm 158.2^{\mathrm{j}, \mathrm{k}}$ \\
\hline Milks (g/day) & $204.5 \pm 119.6$ & $171.1 \pm 128.4$ & $171.0 \pm 123.3$ & $191.8 \pm 136.1$ \\
\hline Cheeses (g/day) & $16.3 \pm 19.0$ & $19.3 \pm 19.8$ & $17.9 \pm 20.5$ & $14.6 \pm 19.0$ \\
\hline Yogurt and fermented milk (g/day) & $58.9 \pm 68.7$ & $43.5 \pm 79.8$ & $43.7 \pm 55.9$ & $61.6 \pm 73.2$ \\
\hline Other dairy products (g/day) & $45.5 \pm 71.7$ & $27.5 \pm 48.0$ & $16.0 \pm 35.3$ & $9.3 \pm 23.2$ \\
\hline Fish and shellfish (g/day) & $44.8 \pm 47.2^{1, \mathrm{~m}}$ & $40.2 \pm 62.8^{\mathrm{m}}$ & $63.7 \pm 72.1^{1, \mathrm{n}}$ & $72.5 \pm 67.7^{\mathrm{n}}$ \\
\hline Meat and meat products (g/day) & $132.5 \pm 61.0^{\circ}$ & $135.7 \pm 71.4^{\circ}$ & $129.0 \pm 90.2^{\circ}$ & $102.7 \pm 60.8^{p}$ \\
\hline Meat (g/day) & $81.8 \pm 54.5$ & $89.5 \pm 65.1$ & $92.7 \pm 67.3$ & $77.1 \pm 55.0$ \\
\hline Sausages and other meat products (g/day) & $50.2 \pm 4.6$ & $45.2 \pm 7.7$ & $35.2 \pm 6.8$ & $23.1 \pm 12.9$ \\
\hline Viscera and spoils (g/day) & $0.5 \pm 4.6$ & $0.9 \pm 7.7$ & $1.2 \pm 6.8$ & $2.5 \pm 12.9$ \\
\hline Eggs (g/day) & $26.1 \pm 22.5$ & $25.0 \pm 29.4$ & $25.6 \pm 24.9$ & $30.4 \pm 28.9$ \\
\hline Pulses (g/day) & $13.8 \pm 13.5$ & $12.2 \pm 17.7$ & $12.5 \pm 16.8$ & $13.3 \pm 17.0$ \\
\hline Sugar and sweets (g/day) & $24.8 \pm 18.2 \mathrm{q}$ & $24.0 \pm 22.4 \mathrm{q}$ & $16.3 \pm 15.5^{\mathrm{r}}$ & $12.0 \pm 13.4^{\mathrm{s}}$ \\
\hline Sugar (g/day) & $2.6 \pm 5.4$ & $3.4 \pm 6.1$ & $6.9 \pm 8.7$ & $5.2 \pm 7.8$ \\
\hline Chocolates (g/day) & $19.0 \pm 17.0$ & $17.2 \pm 17.1$ & $6.1 \pm 9.7$ & $1.2 \pm 4.0$ \\
\hline Jams and other (g/day) & $1.6 \pm 4.5$ & $1.4 \pm 4.4$ & $2.5 \pm 6.9$ & $5.4 \pm 10.8$ \\
\hline Other sweets (g/day) & $1.7 \pm 4.0$ & $2.1 \pm 8.2$ & $0.8 \pm 4.3$ & $0.2 \pm 0.8$ \\
\hline Appetizers (g/day) & $5.2 \pm 10.7$ & $6.2 \pm 12.9$ & $5.3 \pm 12.0$ & $2.6 \pm 9.6$ \\
\hline Ready-to-eat meals (g/day) & $74.4 \pm 78.2^{\mathrm{t}}$ & $74.1 \pm 72.5^{\mathrm{t}, \mathrm{u}}$ & $61.4 \pm 66.4^{\mathrm{t}, \mathrm{u}}$ & $45.5 \pm 58.6^{\mathrm{u}}$ \\
\hline Sauces and condiments (g/day) & $17.9 \pm 21.5^{\mathrm{v}}$ & $14.3 \pm 16.8^{\mathrm{v}}$ & $11.8 \pm 13.4^{\mathrm{w}}$ & $6.1 \pm 8.8^{\mathrm{x}}$ \\
\hline Non-alcoholic beverages (g/day) & $677.9 \pm 367.6^{y}$ & $672.3 \pm 301.6^{y}$ & $886.0 \pm 510.6^{z}$ & $755.7 \pm 435.2 \mathrm{y}, \mathrm{z}$ \\
\hline Water (g/day) & $472.3 \pm 366.3$ & $389.1 \pm 299.1$ & $597.9 \pm 498.6$ & $537.7 \pm 422.9$ \\
\hline Coffee and infusions (g/day) & $6.1 \pm 23.6$ & $8.1 \pm 24.7$ & $101.2 \pm 115.7$ & $133.9 \pm 193.5$ \\
\hline Sugar soft drinks (g/day) & $63.0 \pm 102.3$ & $135.7 \pm 130.8$ & $76.4 \pm 130.4$ & $23.0 \pm 63.8$ \\
\hline Non-sweetened soft drinks (g/day) & $19.6 \pm 59.7$ & $18.6 \pm 79.9$ & $46.7 \pm 130.9$ & $17.9 \pm 63.7$ \\
\hline Sports Drinks (g/day) & $4.3 \pm 28.3$ & $3.3 \pm 23.7$ & $3.3 \pm 25.7$ & $0.6 \pm 6.4$ \\
\hline Juices and nectars (g/day) & $111.2 \pm 124.6$ & $108.8 \pm 165.4$ & $40.4 \pm 69.8$ & $31.9 \pm 77.0$ \\
\hline Other drinks (non-alcoholic) (g/day) & $0.0 \pm 0.0$ & $8.8 \pm 45.7$ & $19.0 \pm 59.5$ & $10.6 \pm 46.1$ \\
\hline Energy drinks (g/day) & $1.3 \pm 11.8$ & $0.0 \pm 0.0$ & $1.0 \pm 11.5$ & $0.0 \pm 0.0$ \\
\hline Alcoholic beverages (g/day) & $0.0 \pm 0.0^{\alpha}$ & $2.9 \pm 2.3^{\alpha}$ & $70.6 \pm 4.8^{\beta}$ & $52.8 \pm 9.8^{\beta}$ \\
\hline High alcohol content beverage (g/day) & $0.0 \pm 0.0$ & $0.0 \pm 0.0$ & $1.9 \pm 12.9$ & $0.0 \pm 0.0$ \\
\hline Low alcohol content beverage (g/day) & $0.0 \pm 0.0$ & $2.9 \pm 20.2$ & $68.8 \pm 140.6$ & $52.8 \pm 101.1$ \\
\hline Supplements and meal replacements (g/day) & $0.0 \pm 0.0$ & $0.0 \pm 0.0$ & $0.3 \pm 4.3$ & $0.1 \pm 0.4$ \\
\hline
\end{tabular}

Data reported as means \pm standard deviation (SD) per group. Different superscript lowercase letters indicate statistical significance in each row for food groups ( $p \leq 0.05$; Bonferroni test).

\section{Discussion}

Dietary habits and food consumption patterns from the population are continuously changing and linked to the evolution of lifestyles and food availability. It is important to emphasize that there has been a rapid evolution in food patterns and, in the last decades, this has been reflected in the mortality and morbidity patterns amongst developed countries, which in turn translates them into a major public health problem, Spain being no exception [14]. At present, it is widely acknowledged that a suboptimal diet is an important preventable risk factor for NCDs. In fact, the Global Burden of Disease collaborators estimated in 2017 that low intake of whole grains (3 million deaths [2-4]) and low intake of fruits (2 million deaths [1-4]) were amongst the leading dietary risk factors for 
deaths worldwide [10]. In addition, it has been projected that by 2030, approximately $69 \%$ of all deaths worldwide will be attributable to NCDs [15]. The influence of dietary intakes as a modifiable risk factor has therefore raised a great interest for food consumption studies, as they represent the first step toward developing feasible prevention strategies [10]. Specifically, over the last three decades, food, work, and home environments have experienced numerous and determinant changes that influenced dietary patterns and food consumption amongst Spanish society [16,17]. Overweight and/or obesity are currently affecting more than $50 \%$ of adults and $25 \%$ of the infant/young population, thus it seems clear that these changes in dietary patterns and lifestyles will have negative consequences for both the present and future generations [18]. Therefore, we have analyzed and described the current food patterns from the Spanish ANIBES study population, being conscious of the difficulties in achieving accurate knowledge of dietary intakes.

The concept of the "Mediterranean Diet" emerged in the 50s with the aim of teaching the population about the dietary patterns and lifestyle of the countries surrounding the Mediterranean Sea [19] which, historically, have been associated with good health and quality of life [8]. The Mediterranean diet is characterized by the abundance of vegetable products (fruits, vegetables, cereals-especially whole grains-nuts, and legumes); the consumption of olive oil as the main source of dietary fat; a moderate intake of wine and other fermented beverages in meals; a frequent consumption of fresh fish, a moderate intake of dairy products (e.g., yogurt and certain cheeses); white meats; and eggs and low consumption in frequency and quantity of red meats and meat products (sausages and processed products) $[8,20,21]$. However, although Spain is bathed by the Mediterranean Sea, several studies indicate that over the last decades, the adherence of the Spanish population to this food pattern has decreased [16,22-27]. Particularly, the non-alcoholic beverages group has greatly evolved towards increased consumption, and a trend also greatly impacting on the global diet. A systematic review including 65 studies of beverage consumption, conducted between 2000 and 2013 and across different age groups, reported that total beverage intake was in the range of $0.6-1.8 \mathrm{~L}$ per day for children, $0.8-2.0 \mathrm{~L}$ per day for teenagers, and $0.8-3.4 \mathrm{~L}$ per day for adults, and water consumption accounted for $58 \%, 75 \%$, and $80 \%$ of total fluid intake, respectively [28]. Moreover, a recent cross-national analysis of 75 countries has shown that sweetened soft drink consumption globally has increased from $36 \mathrm{~L}$ per person per year in 1997 to $43 \mathrm{~L}$ in 2010 [29]. In Spain, the evolution of non-alcoholic beverage consumption has experienced a great growth in recent decades, which has led to an increase of $27 \%$ in the consumption of non-alcoholic beverages per day from 2000 to the year 2008 [22]. Conversely, a moderate decrease in alcoholic beverages consumption is being observed in the Spanish adult population over the last decades, although present intake should be still considered a public health concern, especially for men. In addition, a shift toward a higher beer consumption instead of the more traditional Mediterranean wine has recently occurred [22]. Other studies mainly emphasize the contribution of beverages to energy and nutrient intakes $[30,31]$ and how the increased beverage consumption has affected diet quality, namely, the caloric profile. Beverage consumption is a major contributor to daily energy intake, but it is often overlooked by individuals as a part of their dietary intakes [32]. Indeed, the types of drinks and their frequency of consumption might have either a negative or a positive impact in our overall nutrient intake. At present, the average energy consumption of the Spanish population in the ANIBES study is $1810 \mathrm{kcal} / \mathrm{day}$, of which $12 \%$ are delivered by beverages. Water was the most consumed beverage, followed by milk, and the contribution of caloric soft drinks to the energy intake was $2 \%$ [13]. However, other population groups from France or Italy show a lower proportion of energy provided by beverages ( $8 \%$ and $6 \%$, respectively) [33,34]. These are very close to the quantities suggested by international authorities who recommended that no more than $10 \%$ of the daily calorie intakes should come from beverages other than water $[35,36]$.

The second most consumed group by the population of the ANIBES study are milk and dairy products. Still, despite this fact, it has experienced a drastic reduction in recent years. In the year 2000, consumption was $416 \mathrm{~g} /$ day [22] while the current consumption is $257 \mathrm{~g} / \mathrm{day}$, which shows a reduction by almost half. The data profile is pretty similar to those obtained from the Household 
Consumption Survey (Ministry of Agriculture, Fisheries and Food) [37] which shows a continuous fall in the consumption of dairy products, mainly for fluid milk, as their consumption in Spanish households in the year 2010 was 5246 tons versus 4937 tons in the year 2017. In this regard, it is relevant to underline that proteins from milk products are of high biological value and that they also provide other nutrients with beneficial effects on certain chronic diseases (fats, carbohydrates, and vitamins and minerals such as calcium and phosphorus) [38]. Furthermore, when we compare milk and dairy product intakes with other European countries, we can observe that populations from the Netherlands has a higher consumption (about $319 \mathrm{~g} /$ day, excluding cheese) than the population in Spain (257.2 g/day) and Belgium (160 g/day) [39,40].

Consumption of vegetables and greens, including potatoes, remained largely unchanged from the year $2000(300 \mathrm{~g} / \mathrm{d})$ to $2008(327 \mathrm{~g} / \mathrm{d})$ and fruits from the year 2000 ( $278 \mathrm{~g} /$ day) to 2008 (305 g/day) [22]. In turn, vegetable and fruit consumption amongst the ANIBES population shows a reduction to $178 \mathrm{~g} /$ day and $158 \mathrm{~g} /$ day, respectively. These data greatly differ from dietary recommendations for the Spanish population, specifically, those by the Spanish Society of Community Nutrition (SENC) [41] that endorse a minimum consumption of vegetables of at least $300 \mathrm{~g} /$ day ( 2 servings) and up to $400 \mathrm{~g} /$ day ( 3 servings) of fruits. These recommendations are based on the strong evidence that relates this food group with cardiovascular disease protection; possible evidence for decreased risk of colon cancer, pancreatic diseases, hip fracture, stroke, depression, and pancreatic diseases [42]. When assessing the number of servings of fruits and vegetables across the Spanish population, a report from the Spanish Nutrition Foundation (FEN) showed that the Spanish population is consuming roughly above 2 servings/day versus the recommended 5 servings/day [43]. When compared with other European countries we may observe that vegetable and fruit intakes in Dutch [39] and Belgian [40] populations are similar but lower than in Italy where vegetable consumption reaches $244 \mathrm{~g} /$ day and fruit $239.6 \mathrm{~g} /$ day [44].

Historically, carbohydrate intakes within the Spanish diet came mainly from grain and derivatives, with bread as the main contributor [5]. In Spain, the evolution of global consumption of the cereals and derivatives group has undergone only a small variation from year 2000 to present [22]. However, if we compare this consumption data with previous decades, a marked decrease in consumption occurred, since in 1964 it was 436 g/person per day in Spanish households, according to the "Family Budget Survey" carried out by the National Institute of Statistics in 1991 [24]. Moreover, the European average in terms of annual consumption of cereals is higher than the Spanish average, around 2.7 kilos per person, with Ireland, Sweden, and Finland being the countries with the highest consumption (above $7 \mathrm{~kg}$ per person) [45]. This decrease in the consumption of cereals and derivatives may be due to the rapid evolution of dietary habits along with the abandonment of "basic foods" replaced by other groups that are more processed and transformed [46]. In addition, the lower consumption of bread in our country has been related to a misperception that it might increase weight, but also a lower quality of bread.

It is also worth highlighting that in Spain there has been a moderate decrease in the consumption of meat and derivatives in the past few years. In line with data from the Food Panel in the year 2000 [22], meat and derivatives intakes were close to $180 \mathrm{~g} /$ day while, at present, results from the ANIBES study show that average daily consumption is near to $146 \mathrm{~g} /$ day, and they are the main protein sources for the whole population (33.1\%) [5]. Nevertheless, mean intake of red meat by the ANIBES population was still higher than the maximum of 3 weekly servings which are being recommended by the SENC Dietary Guidelines [41]. The latter should be also considered within the latest guidelines published by the World Health Organization (WHO), suggesting to avoid high intakes associated with potential deleterious health effects [47]. Moreover, these intakes unquestionably must be considered as high according to the traditional Mediterranean dietary patterns. Other relevant protein sources are fish and shellfish, as an important number of studies have underlined the role of fish intake in the primary and secondary prevention of cardiovascular disease, mainly because of their high content in long-chain n-3 polyunsaturated fatty acids [48,49]. Fish and shellfish are also a good source of key nutrients 
such as vitamin D, A, and calcium [50,51]. However, their consumption has decreased approximately $30 \%$ in the past few years in Spain and mainly for the youngest; in the year 2000, average intakes amongst the Spanish population were $88.9 \mathrm{~g} /$ day [22] and at present are about $62.0 \mathrm{~g} /$ day per person, which, in any case, represent a higher consumption than the observed amongst the Italian population (33.7 g/day) [44] and, still, Spain retains the second highest world consumption.

Likewise, eggs, which are an optimal source of unsaturated fats, high-quality protein, folates, and many vitamins and minerals [52], have experienced a decrease from observed intakes in the year 2000, which were 4.3 medium-sized eggs per week [22,25]. Probably, this was a consequence of bad press received health wise, associated to their high cholesterol content. Our results showed egg intakes of $29.3 \mathrm{~g} /$ day, significantly higher in men ( $32.8 \mathrm{~g} /$ day), which is in agreement with previous results from the Food Consumption Survey—32 g/day [25]—still low compared to intakes from the year 2000. Nonetheless, the latest Dietary Guidelines for the Spanish population [41] feature that the consumption of 3 to 5 egg units per week may represent a good nutritional alternative to meat and fish products. This recommendation encourages the increase in egg consumption as a nutritious food source.

Oils and fats represented the main sources of lipids for the Spanish population of which, fortunately, olive oil accounted for $36.9 \%$ of the monounsaturated fatty acid intakes [5]. The consumption of oils and fats by the ANIBES population was $25 \mathrm{~g} /$ day, which shows a considerable reduction in this group intake, since in the year 2000 their consumption was $49.2 \mathrm{~g} /$ day. Fats and oils intakes amongst the Dutch and Belgian populations are quite comparable to the consumption by the Spanish population but is lower than the one observed amongst Italians (33.1 g/day) [44]. It is worth underlining that the consumption of olive oil by the ANIBES population was $17.5 \mathrm{~g} /$ day, which reflects that although the total intake of this food group has been reduced, there is a predilection for this culinary fat as a key dietary component of Mediterranean countries. Olive oil is at the core of the Mediterranean diet and it is the best fat source, as it has been recognized for centuries for its nutritional quality and health associated effects $[39,53]$. Other fat sources, such as sunflower and vegetable-refined oils, represent a minor percentage of the ANIBES population consumption ( $4.4 \pm 5.2 \mathrm{~g} /$ day). Moreover, butter and margarine consumption were even lower $(3.5 \pm 6.2 \mathrm{~g} /$ day $)$ when compared with intakes from other countries such as the United Kingdom and Germany [54,55]. The latter might be explained in part as these are not mainly used as cooking fats or added to food dishes as salads, where Spain still prioritizes olive oil—in fact, the Mediterranean food legacy.

Legumes and pulses are one of the food groups for which intakes have negatively decreased in the last decades in Spain. Specifically, in the year 1964, their average consumption was about $41 \mathrm{~g} /$ day [27] and at present it is only $13 \mathrm{~g} /$ day. One goal of the Spanish SENC guidelines [41] is to increase pulse consumption by encouraging people to eat a 60 to $80 \mathrm{~g}$ serving at least two to four times per week (versus roughly 1 serving size per week at present), since these foods are a primary source of protein, fiber, and other essential nutrients that may have a potential role in colon cancer prevention and reduction of cholesterol levels, as well as being a key element for a better adherence to the Mediterranean dietary pattern.

Interestingly, our analysis demonstrates important differences in food consumption patterns between men and women. Men consumed more cereals and derivatives, meat and meat products, eggs, ready-to-eat meals, sauces and condiments, and alcoholic beverages than women did, whereas women consumed more fruits. These findings are generally consistent with findings from other studies [56-58]. A possible explanation for this might be that women are frequently more weight-conscious and believe that it may be advisable to limit carbohydrates and fat consumption [59]. In addition, they usually have healthier eating habits than men [60]. Likewise, age has a strong influence in food selection, as our results show that children and young individuals consume a higher proportion of cereals and derivatives, milk and dairy products, sugar and sweets, and ready-to-eat meals, while adults have higher vegetable, fruit, and fish and shellfish intakes. Several studies support these findings, showing that consumption of fruit and vegetables generally increase during the transition of the population from a young to adult age, while intakes of cereals and milk and dairy products tend 
to decrease [40]. In the United Kingdom, Lake et al. [61] reported that food group intakes changed considerably between adolescence and adulthood. In this longitudinal study conducted from 1980 to 2000, with those between the ages of 12 and 33 y, consumption of foods containing fat and/or sugar and milk and dairy foods decreased, while intakes of fruits and vegetables increased. Intakes of bread, other cereals and potatoes; fruits and vegetables and meat; and fish and alternatives remained constant from adolescence to adulthood. The authors noted that although adolescent eating patterns are commonly described as unhealthy, in their study sample, some dietary patterns were worth maintaining to achieve a healthy diet at an older age. These facts underline the importance of acquiring and educating healthy eating patterns, culinary skills, and gastronomic experiences in childhood and adolescence ages.

Strengths and Limitations of the Present Study

The strengths of the study include the careful design, protocol, and methodology; the use of a representative sample of the Spanish population aged 9 to 75 years; the innovative methodology for compiling dietary data and dietary assessment method; as well as the extended and updated food database created that allowed to obtain data not only from major food groups but also subgroups of key interest. A limitation of this study is its cross-sectional design, which provides evidence for associations but not for causal relationships. Another type of limitation to consider is what food patterns were established based on a 3 day meal record and whether it is possible that the foods occasionally consumed were not properly valued.

\section{Conclusions}

The present study showed that the majority of the Spanish population, mainly the younger groups, is moving away from the traditional Mediterranean dietary pattern, compared to a few decades ago. The results obtained according to different ages, gender, and for different food groups and subgroups, reinforce the idea that tailored food and nutrition policies are urgently needed.

Author Contributions: T.P., M.d.L.S.-V. and E.R. analyzed the data, contributed to the design of the manuscript and to the interpretation and discussion of the results. J.A.-B., Á.G., M.G.-G., R.M.O. and L.S.-M. are members of the Scientific Advisory Board of the ANIBES study. These authors were responsible for the careful review of the study protocol, design and methodology, providing scientific advice to the study and for the interpretation of the results. They also critically reviewed the manuscript. G.V.-M., the Principal Investigator, was responsible for the design, protocol, methodology, and follow-up/checking of the study. G.V.-M. also revised the manuscript. All authors approved the final version of the manuscript.

Funding: The study was financially supported by a grant from Coca-Cola Iberia through an agreement with the Spanish Nutrition Foundation (Fundación Española de la Nutrición (FEN). The funding sponsor had no role in the design of the study, the collection, analysis nor interpretation of the data, the writing of the manuscript, or in the decision to publish the results.

Acknowledgments: The authors would like to thank Coca-Cola Iberia and IPSOS for its support and technical advice, particularly Rafael Urrialde and Javier Ruiz.

Conflicts of Interest: The authors declare no conflict of interest.

\section{References}

1. Kontogianni, M.D.; Vidra, N.; Farmaki, A.E.; Koinaki, S.; Belogianni, K.; Sofrona, S.; Magkanari, F.; Yannakoulia, M. Adherence rates to the Mediterranean diet are low in a representative sample of Greek children and adolescents. J. Nutr. 2008, 138, 1951-1956. [CrossRef] [PubMed]

2. Park, Y.; Subar, A.F.; Hollenbeck, A.; Schatzkin, A. Dietary fiber intake and mortality in the NIH-AARP diet and health study. Arch. Intern. Med. 2011, 171, 1061-1068. [CrossRef] [PubMed]

3. Uranga, J.A.; López-Miranda, V.; Lombó, F.; Abalo, R. Food, nutrients and nutraceuticals affecting the course of inflammatory bowel disease. Pharmacol. Rep. 2016, 68, 816-826. [CrossRef] [PubMed]

4. Statovci, D.; Aguilera, M.; MacSharry, J.; Melgar, S. The Impact of Western Diet and Nutrients on the Microbiota and Immune Response at Mucosal Interfaces. Front. Immunol. 2017, 8, 838. [CrossRef] [PubMed] 
5. Ruiz, E.; Ávila, J.M.; Valero, T.; Del Pozo, S.; Rodriguez, P.; Aranceta-Bartrina, J.; Gil, A.; González-Gross, M.; Ortega, R.M.; Serra-Majem, L.; et al. Energy Intake, Profile, and Dietary Sources in the Spanish Population: Findings of the ANIBES Study. Nutrient 2015, 7, 4739-4762. [CrossRef]

6. Aranceta, J.; Pérez-Rodrigo, C.; Ribas, L.; Serra-Majem, L.; Serra-Majem, L. Sociodemographic and lifestyle determinants of food patterns in Spanish children and adolescents: The enKid study. Eur. J. Clin. Nutr. 2003, 57, S40-S44. [CrossRef] [PubMed]

7. Pérez-Farinós, N.; López-Sobaler, A.M.; Dal Re, M.; Villar, C.; Labrado, E.; Robledo, T.; Ortega, R.M. The ALADINO study: A national study of prevalence of overweight and obesity in Spanish children in 2011. BioMed Res. Int. 2013, 2013, 163687. [CrossRef]

8. Willett, W.C.; Sacks, F.; Trichopoulou, A.; Drescher, G.; Ferro-Luzzi, A.; Helsing, E.; Trichopoulos, D. Mediterranean diet pyramid: A cultural model for healthy eating. Am. J. Clin. Nutr. 1995, 61, 1402-1406. [CrossRef]

9. Martínez-González, M.A.; Salas-Salvadó, J.; Estruch, R.; Corella, D.; Fitó, M.; Ros, E.; Predimed Investigators. Benefits of the Mediterranean Diet: Insights from the PREDIMED Study. Prog. Cardiovasc. Dis. 2015, 58, $50-60$.

10. GBD Collaborators. Health effects of dietary risks in 195 countries, 1990-2017: A systematic analysis for the Global Burden of Disease Study. Lancet 2019, 393, 1958-1972. [CrossRef]

11. Micha, R.; Khatibzadeh, S.; Shi, P.; Andrews, K.G.; Engell, R.E.; Mozaffarian, D.; Ezzati, M.; Fahimi, S.; Powles, J.; Byers, T.E.; et al. Global, regional and national consumption of major food groups in 1990 and 2010: A systematic analysis including 266 country-specific nutrition surveys worldwide. BMJ Open 2015, 5, e008705. [CrossRef] [PubMed]

12. Ruiz, E.; Ávila, J.M.; Castillo, A.; Valero, T.; del Pozo, S.; Rodriguez, P.; Bartrina, J.A.; Gil, A.; González-Gross, M.; Ortega, R.M.; et al. The ANIBES Study on Energy Balance in Spain: Design, Protocol and Methodology. Nutrients 2015, 7, 970-998. [CrossRef] [PubMed]

13. Nissensohn, M.; Sánchez-Villegas, A.; Ortega, R.M.; Aranceta-Bartrina, J.; Gil, A.; González-Gross, M.; Varela-Moreiras, G.; Serra-Majem, L. Beverage Consumption Habits and Association with Total Water and Energy Intakes in the Spanish Population: Findings of the ANIBES Study. Nutrients 2016, 8, 232. [CrossRef]

14. World Health Organization. Noncommunicable Diseases; World Health Organization: Geneva, Switzerland, 2018.

15. Mathers, C.D.; Loncar, D. Projections of global mortality and burden of disease from 2002 to 2030. PLoS Med. 2006, 3, e442. [CrossRef]

16. Varela-Moreiras, G.; Ruiz, E.; Valero, T.; Ávila, J.M.; del Pozo, S. The Spanish diet: An update. Nutr. Hosp. 2013, 28, 13-20.

17. Aranceta, J. Spanish food patterns. Public Health Nutr. 2001, 4, 1399-1402. [CrossRef] [PubMed]

18. Pérez Miguelsanz, J.; Cabrera, W.; Varela-Moreiras, G.; Garaulet, M. Distribución regional de la grasa corporal. Uso de técnicas de imagen como herramienta de diagnóstico nutricional. Nutr. Hosp. 2010, 25, 207-223.

19. Keys, A. Mediterranean diet and public health: Personal reflections. Am. J. Clin. Nutr. 1995, 61, 1321-1323. [CrossRef]

20. Davis, C.; Bryan, J.; Hodgson, J.; Murphy, K. Definition of the Mediterranean Diet; a Literature Review. Nutrients 2015, 7, 9139-9153. [CrossRef]

21. D'Alessandro, A.; De Pergola, G. Mediterranean Diet Pyramid: A Proposal for Italian People. Nutrients 2014, 6, 4302-4316. [CrossRef]

22. Del Pozo, S.; García, V.; Cuadrado, C.; Ruiz, E.; Valero, T.; Ávila, J.M.; Varela-Moreiras, G. Valoración Nutricional de la Dieta Española de acuerdo al Panel de Consumo Alimentario; Fundación Española de la Nutrición (FEN): Madrid, Spain, 2012.

23. León-Muñoz, L.M.; Guallar-Castillón, P.; Graciani, A.; López-García, E.; Mesas, A.E.; Aguilera, M.T.; Banegas, J.R.; Rodríguez-Artalejo, F. Adherence to the Mediterranean diet pattern has declined in Spanish adults. J. Nutr. 2012, 142, 1843-1850. [CrossRef] [PubMed]

24. Varela, G.; Moreiras, O.; Carbajal, A.; Campo, M. Encuesta de Presupuestos Familiares 1990-91; Instituto Nacional de Estadística: Madrid, Spain, 1991.

25. Varela-Moreiras, G.; Ávila, J.M.; Cuadrado, C.; Del Pozo, S.; Ruiz, E.; Moreiras, O. Evaluation of food consumption and dietary patterns in Spain by the Food Consumption Survey: Updated information. Eur. J. Clin. Nutr. 2010, 64, S37-S43. [CrossRef] [PubMed] 
26. Varela Moreiras, G.; Ávila Torres, J.M.; Cuadrado Vives, C.; del Pozo de la Calle, S.; Ruiz Moreno, E.; Moreiras Tuny, O. Valoración de la Dieta Española de acuerdo al Panel de Consumo Alimentario; Ministerio de Medio Ambiente y Medio Rural y Marino: Madrid, Spain, 2008.

27. Varela-Mosquera, G.; García Rodríguez, D.; Moreiras, O. La Nutrición de los Españoles: Diagnostico y Recomendaciones; Publicaciones de la Escuela Nacional de Administración Pública: Madrid, Spain, 1971.

28. Özen, A.; Bibiloni, M.d.M.; Pons, A.; Tur, J. Fluid intake from beverages across age groups: A systematic review. J. Hum. Nutr. Diet. 2015, 28, 417-442. [CrossRef] [PubMed]

29. Basu, S.; McKee, M.; Galea, G.; Stuckler, D. Relationship of Soft Drink Consumption to Global Overweight, Obesity, and Diabetes: A Cross-National Analysis of 75 Countries. Am. J. Public Health 2013, 103, 2071-2077. [CrossRef] [PubMed]

30. Kant, A.K.; Graubard, B.I.; Atchison, E.A. Intakes of plain water, moisture in foods and beverages, and total water in the adult US population-Nutritional, meal pattern, and body weight correlates: National Health and Nutrition Examination Surveys 1999-2006. Am. J. Clin. Nutr. 2009, 90, 655-663. [CrossRef]

31. An, R. Plain Water and Sugar-Sweetened Beverage Consumption in Relation to Energy and Nutrient Intake at Full-Service Restaurants. Nutrients 2016, 8, 263. [CrossRef]

32. Safefood. Drinks: Consumer Knowledge and Practice in Relation to Drinks for Children and Young People. Available online: https:/www.safefood.eu/SafeFood/media/SafeFoodLibrary/Documents/Publications/ Research\%20Reports/9650_Drinks_Interior-Cover.pdf (accessed on 15 January 2019).

33. Mistura, L.; D'Addezio, L.; Turrini, A. Beverage Consumption Habits in Italian Population: Association with Total Water Intake and Energy Intake. Nutrients 2016, 8, 674. [CrossRef]

34. Nissensohn, M.; Sánchez-Villegas, A.; Galan, P.; Turrini, A.; Arnault, N.; Mistura, L.; Ortiz-Andrellucchi, A.; de Edelenyi, F.S.; D’Addezio, L.; Serra-Majem, L. Beverage Consumption Habits among the European Population: Association with Total Water and Energy Intakes. Nutrients 2017, 9, 383. [CrossRef]

35. European Food Safety Authority (EFSA). Panel on Dietetic Products, Nutrition, and Allergies (NDA): Scientific Opinion on Dietary reference values for water. EFSA J. 2010, 8, 1459.

36. WHO/FAO (World Health Organization/Food and Agriculture Organization of the United Nations). Diet, Nutrition and the Prevention of Chronic Diseases; World Health Organization: Geneva, Switzerland, 2002; Volume 916.

37. Ministerio de Agricultura, Pesca y Alimentación (MAPA). Panel de Consumo Alimentario. Available online: https://www.mapa.gob.es/es/alimentacion/temas/consumo-y-comercializacion-y-distribucion-alimentaria/ panel-de-consumo-alimentario/ (accessed on 24 May 2019).

38. Huth, P.J.; Park, K.M. Influence of dairy product and milk fat consumption on cardiovascular disease risk: A review of the evidence. Adv. Nutr. 2012, 3, 266-285. [CrossRef]

39. Buckland, G.; Mayén, A.L.; Agudo, A.; Travier, N.; Navarro, C.; Huerta, J.M.; Chirlaque, M.D.; Barricarte, A.; Ardanaz, E.; Moreno-Iribas, C.; et al. Olive oil intake and mortality within the Spanish population (EPIC-Spain). Am. J. Clin. Nutr. 2012, 96, 142-149. [CrossRef]

40. Bel, S.; de Ridder, K.A.A.; Lebacq, T.; Ost, C.; Teppers, E.; Cuypers, K.; Tafforeau, J. Habitual food consumption of the Belgian population in 2014-2015 and adherence to food-based dietary guidelines. Arch. Public Health 2019, 77, 14. [CrossRef] [PubMed]

41. Sociedad Española de Nutrición Comunitaria (SENC). Guía de la Alimentación Saludable Para Atención Primara y Colectivos Ciudadanos; Planeta: España, Spain, 2019.

42. Angelino, D.; Godos, J.; Ghelfi, F.; Tieri, M.; Titta, L.; Lafranconi, A.; Marventano, S.; Alonzo, E.; Gambera, A.; Sciacca, S.; et al. Fruit and vegetable consumption and health outcomes: An umbrella review of observational studies. Int. J. Food Sci. Nutr. 2019, 70, 652-667. [CrossRef] [PubMed]

43. Fundación Española de la Nutrición (FEN). Informe de Estado de Situación Sobre: Frutas y hortalizas: Nutrición y Salud en la España del S. XXI. Available online: https://www.fen.org.es/storage/app/media/ imgPublicaciones/informe_frutas_y_hortalizas_fen_2018-v1.pdf (accessed on 21 June 2019).

44. Pounis, G.; Bonanni, A.; Ruggiero, E.; di Castelnuovo, A.; Costanzo, S.; Persichillo, M.; Bonaccio, M.; Cerletti, C.; Riccardi, G.; Donati, M.B.; et al. Food group consumption in an Italian population using the updated food classification system FoodEx2: Results from the Italian Nutrition \& HEalth Survey (INHES) study. Nutr. Metab. Cardiovasc. Dis. 2017, 27, 307-328. [CrossRef] [PubMed] 
45. Dunstan, D.W.; Kingwell, B.A.; Larsen, R.; Healy, G.N.; Cerin, E.; Hamilton, M.T.; Shaw, J.E.; Bertovic, D.A.; Zimmet, P.Z.; Salmon, J.; et al. Breaking Up Prolonged Sitting Reduces Postprandial Glucose and Insulin Responses. Diabetes Care 2012, 35, 976. [CrossRef] [PubMed]

46. van den Boom, A.; Serra-Majem, L.; Ribas, L.; Ngo, J.; Pérez-Rodrigo, C.; Aranceta, J.; Fletcher, R. The contribution of ready-to-eat cereals to daily nutrient intake and breakfast quality in a Mediterranean setting. J. Am. Coll. Nutr. 2006, 25, 135-143. [CrossRef] [PubMed]

47. Bouvard, V.; Loomis, D.; Guyton, K.Z.; Grosse, Y.; Ghissassi, F.E.; Benbrahim-Tallaa, L.; Guha, N.; Mattock, H.; Straif, K. Carcinogenicity of consumption of red and processed meat. Lancet Oncol. 2015, 16, 1599-1600. [CrossRef]

48. Kris-Etherton, P.M.; Harris, W.S.; Appel, L.J. American Heart Association. Nutrition Committee. Fish consumption, fish oil, omega-3 fatty acids, and cardiovascular disease. Circulation 2002, 106, 2747-2757. [CrossRef]

49. Rimm, E.B.; Appel, L.J.; Chiuve, S.E.; Djoussé, L.; Engler, M.B.; Kris-Etherton, P.M.; Mozaffarian, D.; Siscovick, D.S.; Lichtenstein, A.H. Seafood Long-Chain n-3 Polyunsaturated Fatty Acids and Cardiovascular Disease: A Science Advisory from the American Heart Association. Circulation 2018, 138, e35-e47. [CrossRef]

50. González-Rodríguez, L.; Estaire, P.; Peñas-Ruiz, C.; Ortega, R. Vitamin D intake and dietary sources in a representative sample of Spanish adults. J. Hum. Nutr. Diet. 2013, 26, 64-72. [CrossRef]

51. Olza, J.; Aranceta-Bartrina, J.; González-Gross, M.; Ortega, R.; Serra-Majem, L.; Varela-Moreiras, G.; Gil, Á. Reported Dietary Intake, Disparity between the Reported Consumption and the Level Needed for Adequacy and Food Sources of Calcium, Phosphorus, Magnesium and Vitamin D in the Spanish Population: Findings from the ANIBES Study. Nutrients 2017, 9, 168. [CrossRef]

52. Song, W.O.; Kerver, J.M. Nutritional contribution of eggs to American diets. J. Am. Coll. Nutr. 2000, 19, 556S-562S. [CrossRef] [PubMed]

53. Martínez-González, M.A.; Sánchez-Villegas, A. The emerging role of Mediterranean diets in cardiovascular epidemiology: Monounsaturated fats, olive oil, red wine or the whole pattern? Eur. J. Epidemiol. 2004, 19, 9-13. [CrossRef]

54. Linseisen, J.; A Welch, A.; Ocké, M.; Amiano, P.; Agnoli, C.; Ferrari, P.; Sonestedt, E.; Chajes, V.; Bueno-De-Mesquita, H.B.; Kaaks, R.; et al. Dietary fat intake in the European Prospective Investigation into Cancer and Nutrition: Results from the 24-h dietary recalls. Eur. J. Clin. Nutr. 2009, 63, S61-S80. [CrossRef] [PubMed]

55. Prättälä, R.S.; Groth, M.V.; Oltersdorf, U.S.; Roos, G.M.; Sekula, W.; Tuomainen, H.M. Use of butter and cheese in 10 European countries: A case of contrasting educational differences. Eur. J. Public Health 2003, 13, 124-132. [CrossRef] [PubMed]

56. Patterson, B.H.; Harlan, L.C.; Block, G.; Kahle, L. Food choices of whites, blacks, and Hispanics: Data from the 1987 national health interview survey. Nutr. Cancer 1995, 23, 105-119. [CrossRef] [PubMed]

57. Prättälä, R.; Paalanen, L.; Grinberga, D.; Helasoja, V.; Kasmel, A.; Petkeviciene, J. Gender differences in the consumption of meat, fruit and vegetables are similar in Finland and the Baltic countries. Eur. J. Public Health 2007, 17, 520-525. [CrossRef]

58. Wardle, J.; Haase, A.M.; Steptoe, A.; Nillapun, M.; Jonwutiwes, K.; Bellisle, F. Gender differences in food choice: The contribution of health beliefs and dieting. Ann. Behav. Med. 2004, 27, 107-116. [CrossRef]

59. Davy, S.R.; Benes, B.A.; Driskell, J.A. Sex differences in dieting trends, eating habits, and nutrition beliefs of a group of midwestern college students. J. Am. Diet. Assoc. 2006, 106, 1673-1677. [CrossRef]

60. Fagerli, R.; Wandel, M. Gender Differences in Opinions and Practices with Regard to a "Healthy Diet". Appetite 1999, 32, 171-190. [CrossRef]

61. Lake, A.A.; Mathers, J.C.; Rugg-Gunn, A.J.; Adamson, A.J. Longitudinal change in food habits between adolescence (11-12 years) and adulthood (32-33 years): The ASH30 Study. J. Public Health 2006, 28, 10-16. [CrossRef]

(C) 2019 by the authors. Licensee MDPI, Basel, Switzerland. This article is an open access article distributed under the terms and conditions of the Creative Commons Attribution (CC BY) license (http://creativecommons.org/licenses/by/4.0/). 